\title{
Theory of Mind (ToM) and counterfactuality deficits in schizophrenia: misperception or misinterpretation?
}

\author{
DAVID I. LEITMAN ${ }^{1,2}$, RACHEL ZIWICH ${ }^{1}$, ROEY PASTERNAK ${ }^{1}$ \\ AND DANIEL C. JAVITT T ${ }^{1,2,3 *}$ \\ ${ }^{1}$ Program in Cognitive Neuroscience and Schizophrenia, Nathan S. Kline Institute for Psychiatric Research, \\ Orangeburg, NY, USA $;{ }^{2}$ Program in Cognitive Neuroscience, The City College of the City University of \\ New York, USA $;^{3}$ Departments of Psychiatry and Neuroscience, NYU School of Medicine, USA
}

\begin{abstract}
Background. Theory of Mind (ToM) refers to the ability to infer another person's mental state based upon interactional information. ToM deficits have been suggested to underlie crucial aspects of social interaction failure in disorders such as autism and schizophrenia, although the development of paradigms for demonstrating such deficits remains an ongoing area of research. Recent studies have explored the use of sarcasm perception, in which subjects must infer an individual's sincerity or lack thereof, as a 'real-life' index of ToM ability, and as an index of functioning of specific right hemispheric structures. Sarcastic detection ability has not previously been studied in schizophrenia, although patients have been shown to have deficits in ability to decode emotional information from speech ('affective prosody').
\end{abstract}

Method. Twenty-two schizophrenia patients and 17 control subjects were tested on their ability to detect sarcasm from spoken speech as well as measures of affective prosody and basic pitch perception.

Results. Despite normal overall intelligence, patients performed substantially worse than controls in ability to detect sarcasm $(d=2 \cdot 2)$, showing both decreased sensitivity $\left(A^{\prime}\right)$ in detection of sincerity versus sarcasm and an increased bias $\left(B^{\prime \prime}\right)$ toward sincerity. Correlations across groups revealed significant relationships between impairments in sarcasm recognition, affective prosody and basic pitch perception.

Conclusions. These findings demonstrate substantial deficits in ability to infer an internal subjective state based upon vocal modulation among subjects with schizophrenia. Deficits were related to, but were significantly more severe than, more general forms of prosodic and sensorial misperception, and are consistent with both right hemispheric and 'bottom-up' theories of the disorder.

\section{INTRODUCTION}

Cardinal in schizophrenia psychopathology is communicatory disturbance (Mitchell \& Crow, 2005). This disturbance is multifaceted and can take the form of language disorganization as well as difficulty in decoding affect based upon either visual (i.e. facial) or auditory input. Frith

\footnotetext{
* Address for correspondence: Dr Daniel C. Javitt, Program in Cognitive Neuroscience in Schizophrenia, The Nathan S. Kline Institute for Psychiatric Research, 140 Old Orangeburg Rd, Orangeburg, NY 10962, USA.

(Email: javitt@nki.rfmh.org)
}

(1992) and others have sought to tie these deficits to impairment in Theory of Mind (ToM), or the ability to infer one's own or another's internal mental state based upon behavioral interaction. In Frith's conceptualization, ToMbased impairments stem from a confabulation between one's subjective cognitive representations of reality and objective reality, leading to false beliefs, delusions and, within social interaction, the negation of communicatory cues vital for attitudinal and/or affective signals (Frith, 1992). Others see ToM deficits as 
reflecting more general impairment of core executive functioning ability and negative symptoms (Hardy-Bayle et al. 1994), although such deficits typically remain significant even when controlling for such factors (e.g. Langdon et al. 2002). ToM has become an important focus of current research as it provides a conceptual framework for unification of otherwise diverse elements of the schizophrenia symptomatology.

Classical methods for testing ToM involve the use of scenarios, either in the form of short stories or graphically, through sequential picture sets in which social situations, jokes or ironic intent are conveyed. In these tasks, a patient's comprehension of the scenario and also their insight into the character's beliefs and/ or intentions are assessed. Such studies have shown significant deficits in patients compared to controls in both childhood-onset (Pilowsky et al. 2000) and adult-onset schizophrenia (Mazza et al. 2001; also see review by Brune, $2005 \mathrm{~b}$ ), although the statistical magnitude of such deficits is frequently limited by the complexity of the tasks involved.

Current research in social cognitive processing and schizophrenia has begun to link ToM ability to social dysfunction and global outcome in the illness. Roncone et al. (2002) found that ToM ability using tasks that consisted of false belief scenarios presented through cartoons correlated with global social functioning, while Brune (2005a) found that ToM defects were a predictor of social behavioral abnormalities using the social behavioral scale (Wykes \& Sturt, 1986). Similarly, a retrospective study found that ToM dysfunction in patients has been associated with poor childhood socialization (Schenkel et al. 2005). These studies suggest that ToM processes may significant mediate social cognition and ability within schizophrenia, and that the failure to develop ToM during childhood may significantly impair outcome in later life.

There has been a recent increased interest in attitudinal communication in speech as a more 'real-life' measure of ToM ability (McDonald, 1999; Channon et al. 2005; Shamay-Tsoory et al. 2005), especially communication of sarcastic versus literal intent. Within everyday social interaction, attitudinal communications are frequently made in which the literal content of a series of words is negated by the modulation of stress and intonation. Thus, sentences such as 'Now that was a good idea', when stated with primary stress and typical downward going, sentence-ending intonation on the last word typically convey their literal meaning, whereas the same sentence stated with stress on the second word and absence of usual intonation on the final word convey counterfactual intent. Communicating counterfactual intent in this manner is commonly referred to as 'sarcasm'.

Sarcasm can be distinguished from 'irony' and general pragmatics, in that it communicates criticism often of a particular target, and its prosody also conveys attitudes such as scorn or contempt (Jorgensen, 1996). The comprehension of sarcasm has been directly related to mentalizing ability (Winner \& Leekam, 1991), in that comprehension of counterfactual intent is predicated on one's ability to cognitively represent the mental state of others as well as oneself (McDonald, 1999). More explicitly, Channon et al. (2005) have shown that sarcasm comprehension ability correlates strongly with non-linguistic ToM tasks when presented as written dialogue within a social scenario.

While sarcasm perception deficits have not previously been studied in schizophrenia, patients have been shown to have significant difficulty in decoding other introceptive information, such as affect, from vocal intonation (prosody) (Kerr \& Neale, 1993; Ross et al. 2001; Kee et al. 2003). Given these findings, the present study investigates the degree to which patients with schizophrenia can infer counterfactuality of communication as well. Both affective prosody and sarcasm perception are thought to depend upon right hemisphere $(\mathrm{RH})$ functioning (Kaplan et al. 1990; Ozonoff \& Miller, 1996; Mitchell \& Crow, 2005; ShamayTsoory et al. 2005), with lesions studies suggesting that right prefrontal cortex (rPFC) integrates affective sensory information crucial for sarcasm perception (Shamay-Tsoory et al. 2005). Similarly, ToM function has been linked to medial PFC, the superior temporal sulcus and the temporo-parietal junction (Frith \& Frith, 1999; Apperly et al. 2004). These findings provide a potential neuroanatomical underpinning for the present results.

In prior studies, we have also demonstrated that affective dysprosodia in patients correlates 
Table 1. Demographic and clinical characteristics of healthy control and patient populations (values are mean \pm S.D.)

\begin{tabular}{lcc}
\hline \hline $\begin{array}{l}\text { Demographic/clinical } \\
\text { criteria }\end{array}$ & $\begin{array}{c}\text { Control } \\
(n=17)\end{array}$ & $\begin{array}{c}\text { Schizophrenia } \\
(n=22)\end{array}$ \\
\hline Age (years) & $34 \cdot 8 \pm 8 \cdot 9$ & $37.5 \pm 11 \cdot 2$ \\
Gender (M/F) & $9 / 8$ & $20 / 2$ \\
Verbal IQ & $111 \cdot 5 \pm 10 \cdot 4$ & $96 \cdot 0 \pm 10 \cdot 7$ \\
Illness duration & N.A. & $11 \cdot 4 \pm 2 \cdot 3$ \\
BPRS total scores & N.A. & $43 \cdot 6 \pm 10 \cdot 9$ \\
SANS total scores & N.A. & $31 \cdot 2 \pm 9 \cdot 1$ \\
SANS SS: affective flattening & N.A. & $2 \cdot 0 \pm 0 \cdot 9$ \\
SANS SS: ALOGIA & N.A. & $1 \cdot 6 \pm 0 \cdot 9$ \\
SANS SS: avolition-apathy & N.A. & $2 \cdot 1 \pm 0 \cdot 7$ \\
SANS SS: anhedonia-asociality & N.A. & $2 \cdot 4 \pm 0 \cdot 7$ \\
\hline \hline
\end{tabular}

BPRS, Brief Psychiatric Rating Scale (Overall \& Gorham, 1961); SANS, Scale for the Assessment of Negative Symptoms (Andreasen, 1984); SS, subscales; N.A., not applicable.

with deficits in basic pitch perception such as the ability to match tones following a brief delay, suggesting significant 'bottom-up' contributions to higher order dysfunction as well (Leitman et al. 2005). Given that sarcastic intent utilizes tonal modulation, we tested the ability of subjects to perceive sarcasm in short vocal utterances, in concert with pitch and musical perception. We predicted that patients would show a significant impairment in the ability to detect sarcasm, with the impairment being worst in subjects with the poorest sensory performance, suggesting that misperception may contribute significantly to ToM deficits in schizophrenia.

\section{METHOD}

\section{Participants}

Twenty-two patients meeting DSM-IV criteria for either schizophrenia or schizo-affective disorder took part in this study (Table 1). Our patient sample comprised both in-patients $(n=15)$ and out-patients $(n=7)$. All patients were receiving either typical or atypical antipsychotic medication (mean CPZ dose $=1461 \pm 657$ ). Clinical assessment using the Brief Psychiatric Rating Scale (BPRS; Overall \& Gorham, 1961) and the Scale for the Assessment of Negative Symptoms (SANS; Andreasen, 1984) indicated that our patients are severely ill (see Table 1). Both diagnoses and symptom ratings were conducted by trained clinicians, with diagnosis based on the structured clinical interview (SCID) for the DSM-IV (First et al. 1997) and chart review.

The control group consisted of 17 healthy volunteers. These subjects were either volunteers who responded to a local advertisement or hospital staff and were all screened using the SCID. The procedures conducted were under the supervision of the local Institutional Review Board and all subjects had the procedure explained to them verbally before giving their written informed consent. All subjects spoke English as a first language.

\section{Stimuli}

Sarcasm perception was assessed using the attitudinal subtest (APT) of the Aprosodia Battery (Orbelo et al. 2005). This battery consists of 10 semantically neutral sentences, such as "This looks like a safe boat', that were recorded by a female speaker in both a sincere or sarcastic manner for a total of 20 distinct utterances. These utterances were repeated twice to total 40 stimuli. The stimuli were then randomly played on a $C D$ player in a sound-attenuated room at a conversational hearing level. Subjects were instructed to answer after each sentence whether the speaker was being sincere or sarcastic. If subjects were confused by the instructions, further elaboration of the task instructions, using more commonplace synonyms, was provided. Subjects' scores reflected percent correct (Orbelo et al. 2005). Additionally, scores were divided into percent correct of sincere and sarcastic items.

Thus far the APT has been used to assess attitudinal prosody in geriatric populations and is also currently used (Orbelo et al. 2005) in neurological investigations of individuals with brain damage. In these studies patient groups tend to perform worse than comparison subjects on both affective prosody and the APT. This finding, coupled with the fact that our subjects performed above chance and had good within- and between-group variance with no floor or ceiling effects (see results, Fig. 1), suggests that this test is appropriate for examining individual and group differences in schizophrenia.

Affective prosodic ability was assessed using the Voice Emotion Identification Task (VOICEID; Kerr \& Neale, 1993). This test 


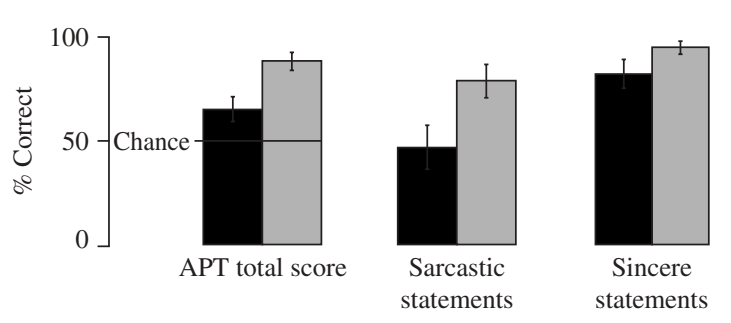

FIG. 1. Per cent correct of sincere (20) and sarcastic (20) statements $(p<0 \cdot 0001)$. Error bars represent S.D. for the mean scores represented in the graph. $\square$, Schizophrenia patients; $\square$, healthy controls.

consists of 21 sentences of neutral content on audiotape. The sentences are spoken by male and female speakers to convey one of six different emotions (happiness, anger, fear, sadness, surprise, or shame). Participants, in a forced choice manner, chose one of the six emotions for identification. Performance is calculated based upon the percentage of correctly identified sentences.

Sensory processing was assessed using two tasks: pure tone matching threshold was obtained using the Tone Matching Task (TMT; Rabinowicz et al. 2000; Leitman et al. 2005), which uses an adaptive 'up-down' transfer staircase method. Three rotating base frequencies of 5000,1000 and $2000 \mathrm{~Hz}$ were used, with tone duration set at $100 \mathrm{~ms}$ and an interstimulus interval between tones of $300 \mathrm{~ms}$. Threshold was assessed as the minimum reliable distinguishable difference between tones. Additionally, the Distorted Tunes Task (DTT; Drayna et al. 2001), a task that has previously shown genetic contributions to pitch perception, was used. This task consists of 26 popular tunes ranging in length from 12 to 26 notes. Seventeen of the tunes are rendered melodically incorrect by changing the pitch of two to nine notes within the tune. Subjects respond 'yes' or 'no' as to whether the melody is correct and are asked to report whether the melody is familiar or not. Patient score reflects the percentage of correctly categorized melodies.

Verbal (pre-morbid) IQ was collected using the quick IQ test (Ammons \& Ammons, 1962). Because of constraints on patient availability, not all measures were collected on all patients. VOICEID and TMT scores have been published previously (Leitman et al. 2005).

\section{Analysis}

Between-group comparisons of sarcasm perception were assessed using an independent sample $t$ test, and effect size calculation and conventions followed Cohen (1988), with a $d$ value of $0.2,0.5$ and 0.8 reflecting the cut-off for small, medium and large effect sizes, respectively. In addition, non-parametric signal detection measures of sensitivity used $A^{\prime}$ after Snodgrass \& Corwin (1988):

$$
\begin{aligned}
A^{\prime}=0 \cdot 5+\left\{\frac{(y-x)(1+y-x)}{4 y(1-x)}\right\} & \text { when } y \geqslant x, \\
\text { or } \quad 0 \cdot 5+\left\{\frac{(x-y)(1+x-y)}{4 x(1-y)}\right\} & \text { when } y \leqslant x
\end{aligned}
$$

and bias $B^{\prime \prime}$ after Grier (1971):

$$
\begin{aligned}
B^{\prime \prime}= & \left\{\frac{y(1-y)-x(1-x)}{y(1-y)+(1-x)}\right\} \quad \text { when } y \geqslant x, \\
& \left\{\frac{x(1-x)-y(1-y)}{x(1-x)+(1-y)}\right\} \quad \text { when } y \leqslant x,
\end{aligned}
$$$$
\text { or }
$$

where $x$ is the probability of falsely identifying a sincere item as sarcastic (False Alarm, FA), and $y$ is the probability of correctly identifying a sarcastic utterance (Hits).

Analysis of covariance (ANCOVA) was used to assess the independence of attitudinal performance for factors of group membership IQ and gender. Logistic regression analysis was used to assess the significance and degree of between-group separation using our combined behavioral measures. In this way we hoped to illustrate the degree of group differentiation possible based on task performance. Correlation analysis of attitudinal perception, prosody and pitch measures was conducted across groups using Pearson's correlation coefficient $(r)$. Correlation analyses of behavioral measures were collected within the patient group using $r$ and Bonferroni corrected for multiple comparisons.

Post hoc analysis examined correlations between symptom ratings and attitudinal prosody. For the BPRS and SANS ratings, correlation analysis used global scores for subscales as well as total scores. All tests were two-tailed with Type I error $\leqslant 0.05$, and statistics were computed in JMP software (SAS Institute Inc., Cary, NC, USA). 
Table 2. Summary of control and patient performance

\begin{tabular}{llrccc}
\hline \hline Measure & \multicolumn{1}{c}{ Group } & $n$ & Mean & s.D. & Effect size $(d)$ \\
\hline Sarcasm & Schizophrenia & 22 & $65 \cdot 6$ & $12 \cdot 2$ & $2 \cdot 2$ \\
& Control & 17 & $88 \cdot 9$ & $8 \cdot 5$ & \\
Sarcasm- $A^{\prime}$ & Schizophrenia & 22 & $0 \cdot 73$ & $0 \cdot 05$ & $1 \cdot 9$ \\
& Control & 17 & $0 \cdot 94$ & $0 \cdot 48$ & \\
Sarcasm- $B^{\prime \prime}$ & Schizophrenia & 22 & $0 \cdot 35$ & $0 \cdot 39$ & $0 \cdot 8$ \\
& Control & 17 & $0 \cdot 68$ & $0 \cdot 45$ & \\
VOICEID & Schizophrenia & 21 & $48 \cdot 3$ & $16 \cdot 8$ & $1 \cdot 6$ \\
& Control & 9 & $71 \cdot 4$ & $10 \cdot 4$ & \multirow{2}{*}{ TMT } \\
& Schizophrenia & 21 & $1 \cdot 01$ & $0 \cdot 57$ & $1 \cdot 3$ \\
& Control & 9 & $0 \cdot 41$ & $0 \cdot 28$ & \\
DTT & Schizophrenia & 20 & $74 \cdot 7$ & $17 \cdot 2$ & $1 \cdot 2$ \\
& Control & 8 & $92 \cdot 7$ & $8 \cdot 0$ & \\
\hline \hline
\end{tabular}

VOICEID, Voice Emotion Identification Test (Kerr \& Neale, 1993); TMT, Tone Matching Threshold (Leitman et al. 2005); DTT, Distorted Tunes Test (Drayna et al. 2001).

\section{RESULTS}

\section{Between-groups analysis}

As predicted, patients showed extremely robust deficits in their ability to perceive sarcasm, as reflected in the total score $[t(37)=-6 \cdot 72$, $p<0 \cdot 0001]$ as well as per cent correct of sarcastic items $[t(37)=-5 \cdot 22, p<0 \cdot 0001]$ recognized and per cent correct of sincere items $[t(37)=-3 \cdot 61$, $p<0.0001]$ (Table 2). Nevertheless, performance was significantly above chance, indicating ability to understand general task demands (Fig. 1). Significant, but smaller, effect size differences between patients and controls for VOICEID $[t(30)=-4 \cdot 12, p<0.0003], \quad$ TMT $[t(30)=3 \cdot 32, p<0 \cdot 002]$ and DTT $[t(29)=-2 \cdot 97$, $p<0.006]$ were also found. Using logistic regression, a metric combining attitudinal prosodic performance and TMT threshold correctly identified $21 / 22$ individuals with schizophrenia $(95 \cdot 4 \%)$, as well as $11 / 12$ controls for whom full data were available $(91 \cdot 7 \%)$.

To further analyze the basis for the deficit, signal detection measures were computed for both sensitivity $\left(A^{\prime}\right)$ and bias $\left(B^{\prime \prime}\right)$. Patients were significantly less sensitive than controls in detecting sarcasm $[t(37)=5 \cdot 14, p<0 \cdot 001]$. Furthermore, patients showed significantly greater bias than controls toward identifying statements as being sincere even when they were not $[t(37)=-2 \cdot 45, p<0 \cdot 02]$. An ANCOVA of attitudinal perception ability controlling for factors of group and IQ and gender revealed that patients' sarcasm deficits were significant overall
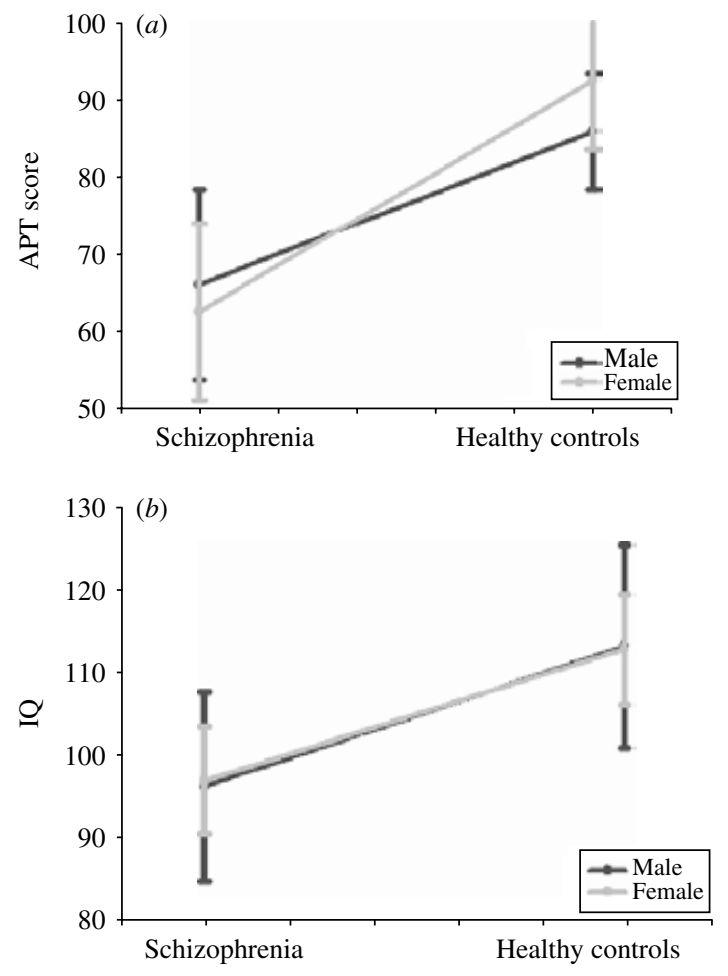

FIG. 2. Gender effects on $(a)$ sarcasm perception and (b) IQ score. Error bars represent S.D. for the mean scores represented in the graph.

$[F(1,28)=16 \cdot 49, p<0 \cdot 0001]$, but that there was a significant IQ effect after controlling for gender differences between groups $[F(1,28)=4 \cdot 36$, $p=0 \cdot 046]$; however, there was no effect of gen$\operatorname{der}[F(1,28)=0.35, p<0 \cdot 56]$ (Fig. 2).

\section{Correlational analysis}

Poor performance on the APT (sarcasm) was significantly correlated with elevated tonematching thresholds $(r=-0 \cdot 39, \mathrm{df}=27, p<$ $0 \cdot 04)$, DTT performance $(r=0 \cdot 40, \quad \mathrm{df}=26$, $p<0.05)$ and poor affective prosodic performance $(r=0.55, \mathrm{df}=27, p<0.003)$ across groups (Fig. 3). Similar patterns of correlation were observed for $A^{\prime}$ [TMT $(r=-0 \cdot 38, \quad \mathrm{df}=27$ $p<0.04)$, DTT $(r=0.43, \mathrm{df}=26, p<0.02)$ and VOICEID $(r=0.56, \mathrm{df}=27, p<0.002)]$, but not $B^{\prime \prime}$. No correlations were found within either group individually (all $p$ 's $>0 \cdot 2$ ). Of the acrossgroups correlations, only the correlations between attitudinal perception and sensitivity 

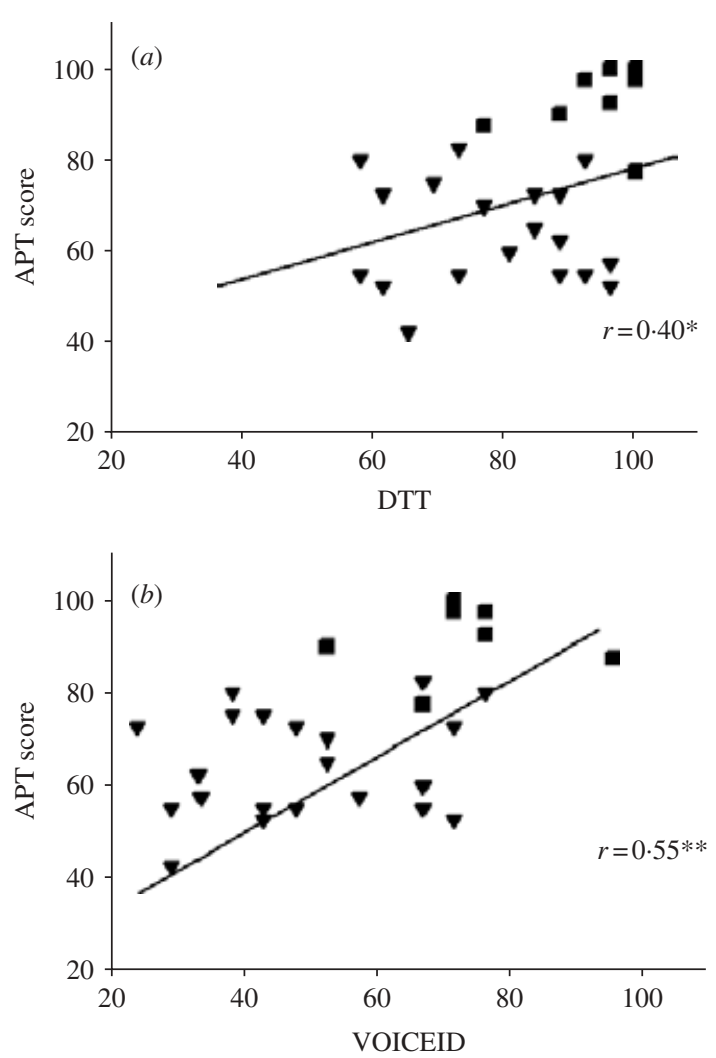

FIG. 3. Scatterplots of (a) Distorted Tunes Test (DTT) and (b) affective prosody Voice Emotion Identification Task (VOICEID) by attitudinal perception score (APT) across groups $(p<0 \cdot 05)$. These figures illustrate in general good separation in performance for both DTT versus APT and VOICEID versus APT. $\square$, Healthy controls; $\boldsymbol{\nabla}$, schizophrenia patients.

$\left(A^{\prime}\right)$ and affective prosody remained significant following Bonferroni correction.

Finally, sarcasm perception scores did not correlate with medication (CPZ) dosage or with positive or negative symptoms as measured by the total scores on the BPRS and SANS, respectively (all $p$ 's $>0 \cdot 1$ ). However, there was a significant correlation between sarcasm perception and the SANS avolition factor $(r=-0 \cdot 44$, $\mathrm{df}=21, p<0 \cdot 03)$.

\section{DISCUSSION}

The primary finding of the present study is that patients show profound deficits in the ability to decode sarcasm based upon tone of voice. The effect size of this deficit ( $d=2 \cdot 2$ s.D. units) was larger than that of affective prosodic deficits observed by ourselves (Leitman et al. 2005) and others (Kerr \& Neale, 1993; Ross et al. 2001), and larger than the 'general' deficit of approximately 1.5 S.D. observed across cognitive domains in schizophrenia (e.g. Bilder et al. 1991; Saykin et al. 1991). Based upon signal detection analyses, this deficit consists of two components - a marked insensitivity to whether statements are sarcastic or sincere $(d=1.9$, $p<0.001$ ), and a less pronounced bias toward accepting statements as sincere even when they were not $(d=0.8, p<0.02)$. Additionally, the results of our ANCOVA indicated that patients have significant sarcasm perception deficits that could not be accounted for solely by IQ or gender differences, although higher IQ did significantly predict better sarcasm perception scores. Thus, our results are somewhat consistent with prior ToM findings (Mazza et al. 2001; Pickup \& Frith, 2001), patients' ability to perceive sarcasm was independent of general intelligence, as reflected by verbal IQ, and may thus represent a specific feature of the disorder. Furthermore, within patients, there were no significant correlations between sarcasm performance and medication dose, suggesting that sarcasm perception is unrelated to antipsychotic treatment.

A significant correlation was observed between sarcasm, affective prosody and musical pitch measures. While no significant correlation was found within the patient group itself, the scatter plot of the across-groups correlation (Fig. 2) reveals that patient and control performance tended to lie along a continuum with control performance and was markedly better than patients on both measures. This finding is consistent with neuropsychological observations that suggest that emotion perception may mediate one's ability to infer counterfactual intent upon which sarcasm comprehension is predicated (Shamay-Tsoory et al. 2005).

The present findings of sarcasm deficits are limited by the fact that they represent a relatively small sample of patients who were primarily male and chronically ill. Furthermore, correlations between our measures of pitch and affective prosody were only significant across groups. Future research must assess whether sarcasm perception impairment is detectable in recent-onset and prodromal schizophrenia 
and whether sarcasm deficits represent a trait of schizophrenia.

Within the framework of ToM, these results can be interpreted in one of several ways. First, deficits in ToM have been linked to dysfunction of rPFC based upon both evaluation of brain lesion (Shamay-Tsoory et al. 2005) and functional imaging (Vollm et al. 2006) approaches. Alternatively, more general RH dysfunction has also been postulated to play a key role in schizophrenia in general (Kaplan et al. 1990; Mitchell \& Crow, 2005). Finally, the ability to decode ToM information in the present task depends heavily on the ability to perceive the relevant intonational cues that communicate counterfactuality. Support for all three possibilities can be found within the body of prior schizophrenia literature, suggesting that the severity of dysfunction in ToM observed using the present paradigm may reflect interactions among multiple overlapping and distributed systems (Vollm et al. 2006).

To the extent that the present deficits may reflect $\mathrm{RH}$ dysfunction, they are supportive of several recent theories proposing RH dysfunction as playing a central role in schizophrenia (e.g. Mitchell \& Crow, 2005). In one study of RH-damaged individuals (Kaplan et al. 1990), subjects were significantly impaired in detecting sarcastic, but not sincere, utterances, similar to that observed in the present study, although signal-detection measures such as sensitivity $\left(A^{\prime}\right)$ and bias $\left(B^{\prime \prime}\right)$ were not reported. Disturbances in $\mathrm{RH}$ function would also be expected to give rise to the disturbances in both verbal (Ross et al. 2001; Leitman et al. 2005) and facial affect recognition (Borod et al. 1989; David \& Cutting, 1990; Heimberg et al. 1992) that have been reported in schizophrenia. Indeed, behavioral studies in schizophrenia (Brune, 2005a), as well as functional magnetic resonance imaging (fMRI) results (Vollm et al. 2006), suggest substantial overlap between general affective cortical networks and specific ToM processing brain regions (Vollm et al. 2006).

In recent years, deficits in basic auditory and visual processing have been extensively documented using both behavioral and neurophysiological paradigms. Patients with schizophrenia show significant deficits in the ability to detect even substantial changes in pitch as measured using both tone matching
(Rabinowicz et al. 2000) and mismatch negativity (MMN) paradigms (Javitt et al. 1995). Within the visual system, patients show significant impairments in functioning of the magnocellular visual pathways, which is crucial for decoding low contrast, low spatial frequency (Butler et al. 2001) and motion information (Kim et al. 2005). Deficits in auditory processing have been shown to contribute significantly in a 'bottom-up' fashion to impairments in affective identification (Leitman et al. 2005).

In the present study, a similar relationship between pitch perception, affective prosody and sarcasm performance was observed, suggesting a substantial bottom-up contribution. A similar relationship has also been observed within the visual system, such that impairment in motion detection ability predicts impaired ability to decode ToM information from visually presented ('eyes test') stimuli (Kelemen et al. 2005). More generally, in mentalizing experiments testing empathy, individuals with greater sensitivity to perceiving affective prosodic cues are also those with greater empathic self-report (Pickett et al. 2004). Thus, while there may be significant dysfunction in the evaluative systems of the brain such as the frontal cortex, a substantial source of ToM and sarcasm performance deficits may be related to the misperception of the cues themselves.

The correlations between patient clinical ratings of negative symptom subscales such as 'avolition' and our ToM measure replicate prior ToM schizophrenia findings and further underscore the theoretical implications for the role of affect in ToM processing mentioned above. Prior research has shown strong correlations between basic sensory deficits and negative symptoms as well as global outcome measures, yet interpretation of these results remained enigmatic.

Like affective prosody (Leitman et al. 2005), impairments in perception of sarcasm may result from significant bottom-up antecedents. Such social communicatory impairment as the inability to recognize affect and attitudinal changes may have profound effects on global outcome in a society in which social communication is a pre-condition for working and living well with others. In this case, inability to recognize sarcasm may lead individuals to feel that they have been misled, rather than just belittled, 
following a counterfactual communication. The connotation of different inflections, such as those used to communicate emotional or attitudinal prosody, is not innate and must be learned during childhood and adolescence. To the extent that sensory dysfunction interferes with such learned associations, it may also undermine the ability to develop ToM concepts, such as counterfactual communication.

In addition to theoretical implications for schizophrenia, the present study also underscores practical issues in clinical communication. Although use of sarcasm is never recommended in clinical communication, it is nevertheless a common feature of societal interaction. The present findings, more even than other ToM studies in schizophrenia, underscore the great difficulty that patients have in decoding counterfactual intent, and in addition demonstrate a bias toward trust that may, in itself, contribute significantly to poor social functioning in schizophrenia. Caregivers in general, however, may be unaware of the profundity of such deficits, given the ease with which sarcasm is detected by most unaffected individuals. The present findings thus not only weigh against use of intonation to convey counterfactuality of information during therapeutic communications in patients with schizophrenia but also suggest that deficits in this real-world ability should be appreciated by family members and other caregivers in order to minimize potential miscommunications that may lead to adverse outcomes in schizophrenia.

\section{ACKNOWLEDGMENTS}

This research was supported in part by NIMH grants NRSA F1-MH067339 to D.I.L. and grants K02 MH01439 and R01 MH49334 and a Translational Research Scientist Award from the Burroughs Welcome Fund to D.C.J. We acknowledge the assistance provided by $\mathrm{Dr}$ Elliot Ross for the use of his Attitudinal Prosody battery, Dr Kerr and Dr Neale for the use of the Voice Emotion Identification task and Dr Denis Drayna for the use of the Distorted Tunes Test.

\section{DECLARATION OF INTEREST}

None.

\section{REFERENCES}

Ammons, R. B. \& Ammons, C. H. (1962). The Quick Test (QT): provisional manual. Psychological Reports 11, 111-162.

Andreasen, N. C. (1984). The Scale for the Assessment of Negative Symptoms (SANS). The University of Iowa: Iowa City.

Apperly, I. A., Samson, D., Chiavarino, C. \& Humphreys, G. W. (2004). Frontal and temporo-parietal lobe contributions to theory of mind: neuropsychological evidence from a false-belief task with reduced language and executive demands. Journal of Cognitive Neuroscience 16, 1773-1784.

Bilder, R. M., Lipschutz-Broch, L., Reiter, G., Geisler, S., Mayerhoff, D. \& Lieberman, J. A. (1991). Neuropsychological deficits in the early course of first episode schizophrenia. Schizophrenia Research 5, 198-199.

Borod, J. C., Alpert, M., Brozgold, A., Martin, C., Welkowitz, J., Diller, L., Peselow, E., Angrist, B. \& Lieberman, A. (1989). A preliminary comparison of flat affect schizophrenics and braindamaged patients on measures of affective processing. Journal of Communication Disorders 22, 93-104.

Brune, M. (2005a). Emotion recognition, 'theory of mind,' and social behavior in schizophrenia. Psychiatry Research 133, 135-147.

Brune, M. (2005b). 'Theory of mind' in schizophrenia: a review of the literature. Schizophrenia Bulletin 31, 21-42.

Butler, P. D., Schechter, I., Zemon, V., Schwartz, S. G., Greenstein, V. C., Gordon, J., Schroeder, C. E. \& Javitt, D. C. (2001) Dysfunction of early-stage visual processing in schizophrenia. American Journal of Psychiatry 158, 1126-1133.

Channon, S., Pellijeff, A. \& Rule, A. (2005). Social cognition after head injury: sarcasm and theory of mind. Brain and Language $\mathbf{9 3}$, $123-134$

Cohen, J. (1988). Statistical Power Analysis for the Behavioral Sciences. Erlbaum Associates: Hilsdale, NJ.

David, A. S. \& Cutting, J. C. (1990). Affect, affective disorder and schizophrenia. A neuropsychological investigation of right hemisphere function. British Journal of Psychiatry 156, 491-495.

Drayna, D., Manichaikul, A., de Lange, M., Snieder, H. \& Spector, T. (2001). Genetic correlates of musical pitch recognition in humans. Science 291, 1969-1972.

First, M. B., Spitzer, R. L., Gibbon, M. \& Williams, J. (1997) Structural Clinical Interview for DSM-IV Axis I Disorders (SCID$I V)$. Biometrics Research Department, New York State Psychiatric Institute: New York.

Frith, C. D. (1992). The Cognitive Neuropsychology of Schizophrenia. Laurence Erlbaum Associates: Hove, UK.

Frith, C. D. \& Frith, U. (1999). Interacting minds - a biological basis Science 286, 1692-1695.

Grier, J. B. (1971). Nonparametric indexes for sensitivity and bias: computing formulas. Psychological Bulletin 75, 424-429.

Hardy-Bayle, M. C., Passerieux, C., Claudel, B., Olivier, V. \& Chevalier, J. F. (1994). Communication disorders in schizophrenic patients. Cognitive explanation and clinical reconsideration. Encephale 20, 393-400.

Heimberg, C., Gur, R. E., Erwin, R. J., Shtasel, D. L. \& Gur, R. C. (1992). Facial emotion discrimination: III. Behavioral findings in schizophrenia. Psychiatry Research 42, 253-265.

Javitt, D. C., Doneshka, P., Grochowski, S. \& Ritter, W. (1995) Impaired mismatch negativity generation reflects widespread dysfunction of working memory in schizophrenia. Archives of General Psychiatry 52, 550-558.

Jorgensen, J. (1996). The functions of sarcastic irony in speech. Journal of Pragmatics 26, 613-634.

Kaplan, J. A., Brownell, H. H., Jacobs, J. R. \& Gardner, H. (1990). The effects of right hemisphere damage on the pragmatic interpretation of conversational remarks. Brain and Language 38, 315-333.

Kee, K. S., Green, M. F., Mintz, J. \& Brekke, J. S. (2003). Is emotion processing a predictor of functional outcome in schizophrenia? Schizophrenia Bulletin 29, 487-497.

Kelemen, O., Erdelyi, R., Pataki, I., Benedek, G., Janka, Z. \& Keri, S. (2005). Theory of mind and motion perception in schizophrenia. Neuropsychology 19, 494-500. 
Kerr, S. L. \& Neale, J. M. (1993). Emotion perception in schizophrenia: specific deficit or further evidence of generalized poor performance? Journal of Abnormal Psychology 102, 312-318.

Kim, D., Zemon, V., Saperstein, A., Butler, P. D. \& Javitt, D. C. (2005). Dysfunction of early-stage visual processing in schizophrenia: harmonic analysis. Schizophrenia Research 76, 55-65.

Langdon, R., Coltheart, M., Ward, P. B. \& Catts, S. V. (2002). Disturbed communication in schizophrenia: the role of poor pragmatics and poor mind-reading. Psychological Medicine 32, 1273-1284.

Leitman, D. I., Foxe, J. J., Butler, P. D., Saperstein, A., Revheim, N. \& Javitt, D. C. (2005). Sensory contributions to impaired prosodic processing in schizophrenia. Biological Psychiatry 58, 56-61.

Mazza, M., De Risio, A., Surian, L., Roncone, R. \& Casacchia, M. (2001). Selective impairments of theory of mind in people with schizophrenia. Schizophrenia Research 47, 299-308.

McDonald, S. (1999). Exploring the process of inference generation in sarcasm: a review of normal and clinical studies. Brain and Language 68, 486-506.

Mitchell, R. L. \& Crow, T. J. (2005). Right hemisphere language functions and schizophrenia: the forgotten hemisphere? Brain $\mathbf{1 2 8}$ 963-978.

Orbelo, D. M., Grim, M. A., Talbott, R. E. \& Ross, E. D. (2005). Impaired comprehension of affective prosody in elderly subjects is not predicted by age-related hearing loss or age-related cognitive decline. Journal of Geriatric Psychiatry and Neurology 18, 25-32.

Overall, J. E. \& Gorham, D. E. (1961). The Brief Psychiatric Rating Scale. Psychological Reports 10, 799-812.

Ozonoff, S. \& Miller, J. N. (1996). An exploration of righthemisphere contributions to the pragmatic impairments of autism. Brain and Language 52, 411-434.

Pickett, C. L., Gardner, W. L. \& Knowles, M. (2004). Getting a cue: the need to belong and enhanced sensitivity to social cues. Personality and Social Psychology Bulletin 30, 1095-1107.

Pickup, G. J. \& Frith, C. D. (2001). Theory of mind impairments in schizophrenia: symptomatology, severity and specificity. Psychological Medicine 31, 207-220.

Pilowsky, T., Yirmiya, N., Arbelle, S. \& Mozes, T. (2000). Theory of mind abilities of children with schizophrenia, children with autism, and normally developing children. Schizophrenia Research $\mathbf{4 2}$ $145-155$.

Rabinowicz, E. F., Silipo, G., Goldman, R. \& Javitt, D. C. (2000). Auditory sensory dysfunction in schizophrenia: imprecision or distractibility? Archives of General Psychiatry 57, 1149-1155.

Roncone, R., Falloon, I. R., Mazza, M., De Risio, A., Pollice, R., Necozione, S., Morosini, P. \& Casacchia, M. (2002). Is theory of mind in schizophrenia more strongly associated with clinical and social functioning than with neurocognitive deficits? Psychopathology 35, 280-288.

Ross, E. D., Orbelo, D. M., Cartwright, J., Hansel, S., Burgard, M., Testa, J. A. \& Buck, R. (2001). Affective-prosodic deficits in schizophrenia: profiles of patients with brain damage and comparison with relation to schizophrenic symptoms. Journal of Neurology, Neurosurgery, and Psychiatry 70, 597-604.

Saykin, A. J., Gur, R. C., Gur, R. E., Mozley, P. D., Mozley, L. H., Resnick, S. M., Kester, D. B. \& Stafiniak, P. (1991). Neuropsychological function in schizophrenia. Selective impairment in memory and learning. Archives of General Psychiatry 48, 618 624.

Schenkel, L. S., Spaulding, W. D. \& Silverstein, S. M. (2005). Poor premorbid social functioning and theory of mind deficit in schizophrenia: evidence of reduced context processing? Journal of Psychiatric Research 39, 499-508.

Shamay-Tsoory, S. G., Tomer, R. \& Aharon-Peretz, J. (2005). The neuroanatomical basis of understanding sarcasm and its relationship to social cognition. Neuropsychology 19, 288-300.

Snodgrass, J. G. \& Corwin, J. (1988). Perceptual identification thresholds for 150 fragmented pictures from the Snodgrass and Vanderwart picture set. Perceptual and Motor Skills 67, 3-36.

Vollm, B. A., Taylor, A. N., Richardson, P., Corcoran, R., Stirling, J., McKie, S., Deakin, J. F. \& Elliott, R. (2006). Neuronal correlates of theory of mind and empathy: a functional magnetic resonance imaging study in a nonverbal task. Neuroimage 29, 90-98.

Winner, E. \& Leekam, S. (1991). Distinguishing irony from deception: understanding the speaker's second-order intention. British Journal of Developmental Psychology 9, 257-270.

Wykes, T. \& Sturt, E. (1986). The measurement of social behaviour in psychiatric patients: an assessment of the reliability and validity of the SBS schedule. British Journal of Psychiatry 148, 1-11. 